\title{
Proceedings of the 2021 ISNR Annual Conference (Virtual): Poster Presentations
}

\author{
Selected Abstracts of Conference Poster Presentations at the 2021 International Society for \\ Neuroregulation and Research (ISNR) 29th Annual Conference, Miami, Florida, USA
}

Citation: International Society for Neurofeedback and Research. (2021). Proceedings of the 2021 ISNR Annual Conference (Virtual): Poster Presentations. NeuroRegulation, 8(4), 220-228. https://doi.org/10.15540/nr.8.4.220

Copyright: @ 2021. ISNR. This is an Open Access article distributed under the terms of the Creative Commons Attribution License (CC-BY).

Using Standardized Weighted Low-Resolution Electromagnetic Tomography (swLORETA) to Analyze the Deep Brain Activity for Healthy Adults and Patients with Major Depressive Disorder

Hong-En Yu and I-Mei Lin

Kaohsiung Medical University, Kaohsiung City, Kaohsiung, Taiwan

Background. The differences in brain activity between patients with major depressive disorder (MDD) and healthy adults have been confirmed by functional magnetic resonance imaging (fMRI), positron emission tomography (PET), and electroencephalography (EEG), especially at the prefrontal lobe, posterior cingulate cortex (PCC). However, the high cost of $\mathrm{AMRI}$ and PET and poor time resolution limited the clinical application. Recently, researchers used high time resolution of standardized weighted low-resolution electromagnetic tomography (swLORETA) to refer to deep brain activity. This study aimed to convert EEG raw signals into swLORETA and explore the differences in deep brain activity between patients with MDD and healthy adults.

Method. BrainMaster equipment with a 19-channel EEG cap was used to collect resting EEG data with eyes closed for $5 \mathrm{~min}$. NeuroGuide software was used to remove the EEG artifacts, and swLORETA software was used to analyze the 12700 voxels of current source density (CSD) for 114 patients with MDD comorbid with anxiety symptoms and 134 healthy adults. The deep brain activity at the frontal lobe and PCC in different frequency bands were analyzed for delta, theta, alpha, and beta.

Results. There were higher activities at prefrontal lobe (dorsal medial prefrontal cortex [dmPFC], ventral medial prefrontal cortex [vmPFC], dorsal lateral prefrontal cortex [dIPFC], ventral lateral prefrontal cortex [VIPFC], orbital frontal cortex [OFC]) and PCC were found in MDD patients compared with healthy adults, especially for lower delta and theta, and higher beta, beta3, and high-beta.

Conclusion. This study indicated that brain hyperactivity at the right prefrontal lobe (dIPFC and vmPFC) and PCC in patients with MDD comorbid with anxiety symptoms, and the dIPFC and PCC were also related to emotion-regulation in MDD. Inhibited high-beta activity or rewarded delta and theta activities at the right frontal lobe and PCC may be a possible neurofeedback protocol for patients with MDD in the future study.

\section{References}

Begić, D., Popović-Knapić, V., Grubišin, J., Kosanović-Rajačić, B., Filipčić, I., Telarović, I., \& Jakovljević, M. (2011). Quantitative electroencephalography in schizophrenia and depression. Psychiatria Danubina, 23(4), 355-362.

Herrington, J. D., Heller, W., Mohanty, A., Engels, A. S., Banich, M. T., Webb, A. G., \& Miller, G. A. (2010). Localization of asymmetric brain function in emotion and depression. Psychophysiology, 47(3), 442-454. https://doi.org/10.1111 /j.1469-8986.2009.00958.x

Koberda, J. L. (2014). Z-score LORETA neurofeedback as a potential therapy in depression/anxiety and cognitive dysfunction. In R. W. Thatcher \& J. F. Lubar (Eds.), Z score neurofeedback: Clinical applications (1st ed., pp. 93-113). Academic Press.

Koo, P. C., Thome, J., Berger, C., Foley, P., \& Hoeppner, J. (2017). Current source density analysis of resting state EEG in depression: A review. Journal of Neural Transmission, 124(1), 109-118. https://doi.org/10.1007/s00702-015-1432-2

Korb, A. S., Cook, I. A., Hunter, A. M., \& Leuchter, A. F. (2008). Brain electrical source differences between depressed subjects and healthy controls. Brain Topography, 21(2), 138146. https://doi.org/10.1007/s10548-008-0070-5

Lubar, J. F., Congedo, M., \& Askew, J. H. (2003). Low-resolution electromagnetic tomography (LORETA) of cerebral activity in chronic depressive disorder. International Journal of Psychophysiology, 49(3), 175-185. https://doi.org/10.1016 IS0167-8760(03)00115-6

Mayberg, H. S. (2003). Modulating dysfunctional limbic-cortical circuits in depression: Towards development of brain-based algorithms for diagnosis and optimized treatment. British Medical Bulletin, 65(1), 193-207. https://doi.org/10.1093/bmb /65.1.193 
Mientus, S., Gallinat, J., Wuebben, Y., Pascual-Marqui, R. D., Mulert, C., Frick, K., Dorn, H., Herrmann, W. M., \& Winterer, G. (2002). Cortical hypoactivation during resting EEG in schizophrenics but not in depressives and schizotypal subjects as revealed by low resolution electromagnetic tomography (LORETA). Psychiatry Research: Neuroimaging, 116(1-2), 95-111. https://doi.org/10.1016/S09254927(02)00043-4

Palmero-Soler, E., Dolan, K., Hadamschek, V., \& Tass, P. A. (2007). swLORETA: A novel approach to robust source localization and synchronization tomography. Physics in Medicine and Biology, 52(7), 1783-1800. https://doi.org /10.1088/0031-9155/52/7/002

Pizzagalli, D. A., Nitschke, J. B., Oakes, T. R., Hendrick, A. M., Horras, K. A., Larson, C. L., Abercrombie, H. C., Schaefer, S. M., Koger, J. V., Benca, R. M., Pascual-Marqui, R. D., \& Davidson, R. J. (2002). Brain electrical tomography in depression: The importance of symptom severity, anxiety, and melancholic features. Biological Psychiatry, 52(2), 73-85. https://doi.org/10.1016/S0006-3223(02)01313-6

\section{Frontal EEG Indices of Attentional Bias and} Involuntary Orienting to Pictorial Drug-related Cues in Cocaine Addiction

Estate Sokhadze ${ }^{1}$, Mohamed Shaban', Ayman El-Baz ${ }^{3}$, and Allan Tasman ${ }^{3}$

${ }^{1}$ University of South Carolina School of Medicine, Greenville, South Carolina, USA

'University of South Alabama, Mobile, Alabama, USA

${ }^{3}$ University of Louisville, Louisville, Kentucky, USA

Background. Preoccupation with drug and drugrelated items is a typical characteristic of addicted individuals. Several research studies provided support for the hypothesis about the alteration of attention processes in chronic addicts (Hester et al., 2006; Robinson \& Berridge, 2001), so-called attentional bias (Franken, 2003), when drug-related cues attain greater salience and motivational significance (Cox et al., 2006). Cue reactivity refers to a phenomenon in which individuals with a history of drug dependence exhibit verbal, physiological, and behavioral responses to cues associated with their preferred substance of abuse (Carter \& Tiffany, 1999). One of the cognitive components of cue reactivity in substance abusers is the preferential allocation of attentional resources for items related to drug use (Lubman et al., 2000).

Methods. This study explored frontal event-related potentials (ERP) and induced $40 \mathrm{~Hz}$-centered gamma oscillation power to investigate differences associated with responses to attended and unattended drug-related cues in a three-category oddball task using neutral, drug-, and stress-related pictorial stimuli (from the IAPS and matched cocaine images). The study was conducted on 14 individuals with cocaine use disorder (CUD, mean age 44.2 years, 6 females) and 9 age- and gender-matched control (CNT, 36.7 years, 4 females) subjects. A 128-channel EGI-Phillips EEG system was used to record ERP and single trial induced gamma oscillations $(30-40 \mathrm{~Hz})$ during the visual threecategory oddball task with three categories (neutral, drug, stress) of affective pictures, when one of the categories was used as a target (attended stimuli, $25 \%$ ) requiring motor response, while images of other categories including drug images served as unattended nontarget rare $(25 \%)$ distracters.

Results and Discussion. Most profound group differences in reactivity to attended and unattended drug-related stimuli were found in the amplitude and latency of frontal $\mathrm{P} 3 \mathrm{a}$ and in the relative power of induced (280-380 ms poststimulus) $40 \mathrm{~Hz}$-centered gamma oscillations. At the frontal regions-ofinterests (ROI, left, midline, right; three EEG sites per ROI) amplitude of P3a ERP component was higher to unattended drug cues in the cocaine use disorder group as compared to controls (e.g., mean across all ROls $3.55 \mathrm{uV}$ in CUD vs. $1.85 \mathrm{uV}$ in controls, $F=8.58, p<.01$ ) and latency was prolonged. Differences in amplitude and latency of P3a in response to attended target drug cues were not significant. Prefrontal induced gamma oscillations showed similar pattern of excessive reactivity to unattended drug cues in CUD (at FPz, $F$ $=55.64, p<.001$ ) but not to attended targets. Above described changes of depended variables are indicative of involuntary orienting of attention to highly salient drug-related cues even when subjects are not instructed to attend to nor to respond to them. In the prior studies we reported about usefulness of application of ERP and evoked oscillation measures as biomarkers of substance use and as neurofeedback treatment outcomes (Horrell et al., 2010; Sokhadze et al., 2008).

Conclusions. We propose that the employed EEG/ERP cue reactivity variables could be used as valuable functional outcome measures in cocaine drug users undergoing behavioral treatment.

\section{References}

Carter, B. L., \& Tiffany, S. T. (1999). Meta-analysis of cuereactivity in addiction research. Addiction, 94, 327-340.

Cox, W. M., Fadardi, J. S., \& Pothos, E. M. (2006). The AddictionStroop Test: Theoretical considerations and procedural recommendations. Psychological Bulletin, 132(3), 443-476. https://doi.org/10.1037/0033-2909.132.3.443

Franken, I. H. (2003). Drug craving and addiction: Integrating psychological and neuropsychopharmacological approaches. Progress in Neuro-psychopharmacology and Biological Psychiatry, 27(4), 563-579. https://doi.org/10.1016/S02785846(03)00081-2

Hester, R., Dixon, V., \& Garavan, H. (2006). A consistent attentional bias for drug-related material in active cocaine users across word and picture versions of the emotional 
Stroop task. Drug and Alcohol Dependence, 81(3), 251-257. https://doi.org/10.1016/j.drugalcdep.2005.07.002

Horrell, T., El-Baz, A., Baruth, J., Tasman, A., Sokhadze, G., Stewart, C., \& Sokhadze, E. (2010). Neurofeedback effects on evoked and induced EEG gamma band reactivity to drugrelated cues in cocaine addiction. Journal of Neurotherapy, 14(3), 195-216. https://doi.org/10.1080 /10874208.2010.501498

Lubman, D. I., Peters, L. A., Mogg, K., Bradley, B. P., \& Deakin, J. F. (2000). Attentional bias for drug cues in opiate dependence. Psychological Medicine, 30(1), 169-175. https://doi.org/10.1017/s0033291799001269

Robinson, T. E., \& Berridge, K. C. (2001). Incentive-sensitization and addiction. Addiction, 96(1), 103-114. https://doi.org /10.1046/j.1360-0443.2001.9611038.x

Sokhadze, E., Singh, S., Stewart, C., Hollifield, M., El-Baz, A., \& Tasman, A. (2008). Attentional bias to drug- and stressrelated pictorial cues in cocaine addiction comorbid with Posttraumatic Stress Disorder. Journal of Neurotherapy, 12(4), 205-225. https://doi.org/10.1080/10874200802502185

\section{Evaluations of Algorithmic Models for Estimations of Current Source Destiny and Electrophysiological Substrates According to LORETA and swLORETA Analyses Kristin Williams}

University of South Carolina- Speech Neuroscience Lab, Columbia, South Carolina, USA

This research examines the mathematical algorithms utilized for electroencephalographic source imaging (ESI) and current source localization. Electrodynamic processes that are evaluated according to source localization analyses and are derived from electroencephalographic assessments are based on the inverse problem. The inverse problem does not have a unique solution as infinite interactions between neuronal generators may yield the same derivation of scalp potentials. Source localization is subject to significant estimation errors of current source density and dipolar sources. The underdetermination of the system influences the significant estimation errors that can arise from small changes in the data related to the three-dimensional montage utilized to derive the estimations of the position and direction of potential electrophysiological generators for the lead field matrix. Common mathematical algorithms proposed to solve the inverse problem include low-resolution electromagnetic tomography (LORETA), standardized weighted low-resolution electromagnetic tomography (swLORETA), minimum norm estimate, and the weighted minimum norm estimate. Statistical evaluations of electrophysiological signals can be applied to evaluate neurocognitive behavior as linear or nonlinear functions. This research specifically evaluates the algorithmic models utilized for source localization according to LORETA and swLORETA analyses. Thus, the proposed models will be based upon linear estimations of cortical and subcortical activity that are etiologically relevant to electrodynamic processes. Because arithmetic models that incorporate priors into estimations of source distribution include the Bayesian framework and penalty function, this research examines the calculus related to estimations of source localization according to these models. The penalty function incorporates unknown source dynamics, the number of sensors utilized in the electrophysiological recording, number of samples across time, and a weighted factor. This algorithm utilizes a least squares regression model to estimate the penalty term as a quadratic function. Estimations of EEG sources according to parametric Bayesian models utilize spatial and neural constraints, evaluations of the current, and physical principles related to wave dispersion to derive estimations of localization. The parametric Bayesian model assumes Gaussian distributions and accounts for random fluctuations of sensor and source space. These mathematical models are also included due to the ability to transform one into the other while maintaining the integrity of their core structures.

\section{References}

Gomez, J. F., \& Thatcher, R. W. (2001). Frequency domain equivalence between potentials and currents using LORETA. International Journal of Neuroscience, 107(3-4), 161-171. https://doi.org/10.3109/00207450109150683

Hassan, M., Dufor, O., Merlet, I., Berrou, C., \& Wendling, F. (2014). EEG source connectivity analysis: From dense array recordings to brain networks. PLOS ONE, 9(8), e105041. https://doi.org/10.1371/journal.pone.0105041

Henson, R. N., Wakeman, D. G., Litvak, V., \& Friston, K. J. (2011). A parametric empirical Bayesian framework for the EEG/MEG Inverse Problem: Generative models for multisubject and multi-modal integration. Frontiers in Human Neuroscience, $\quad 5, \quad 76 . \quad$ https://doi.org/10.3389 /fnhum.2011.00076

Lei, X., Wu, T., \& Valdes-Sosa, P. A. (2015). Incorporating priors for EEG source imaging and connectivity analysis. Frontiers in Neuroscience, 9, 284. https://doi.org/10.3389 /fnins.2015.00284

Mahjoory, K., Nikulin, V. V., Botrel, L., Linkenkaer-Hansen, K., Fato, M. M., \& Haufe, S. (2017). Consistency of EEG source localization and connectivity estimates. Neurolmage, 152, 590-601. https://doi.org/10.1101/071597

Nunez, P., \& Srinivasan, R. (2006). Electric fields and currents in biological tissue. In Electric Fields of the Brain: The Neurophysics of EEG (2nd ed., pp. 147-202). New York, NY: Oxford University Press.

Nunez, M. D., Nunez, P. L., \& Srinivasan, R. (2016). Electroencephalography (EEG): Neurophysics, experimental methods, and signal processing. In $\mathrm{H}$. Ombao, M. Linquist, W. Thompson, \& J. Aston (Eds.) Handbook of neuroimaging data analysis (pp. 175-197). Chapman \& Hall/CRC. Advance online publication. https://doi.org/10.13140 /rg.2.2.12706.63687

Pascual-Marqui, R. D., Michel, C. M., \& Lehmann, D. (1994). Low resolution electromagnetic tomography: $A$ new method for 
localizing electrical activity in the brain. International Journal of Psychophysiology, 18(1), 49-65. https://doi.org/10.1016 10167-8760(84)90014-x

Pascual-Marqui, R. D., Faber, P. L., Kinoshita, T., Kochi, K., Milz, P., Nishida, K., \& Yoshimura, M. (2018). Comparing $E E G / M E G$ neuroimaging methods based on localization error, false positive activity, and false positive connectivity. bioRxiv, 269753. https://doi.org/10.1101/269753

Wagner, M., Fuchs, M., \& Kastner, J. (2004). Evaluation of SLORETA in the presence of noise and multiple sources. Brain Topography, 16(4), 277-280. https://doi.org/10.1023 /b:brat.0000032865.58382.62

\section{Psychophysiological Indices of Attentional Bias Towards Drug-related Pictures in Visual Cue Reactivity Test in Individuals with Opiate Use Disorder Enrolled in Buprenorphine- Maintenance Program \\ Erik Ortiz', Ashley Coleman ${ }^{1}$, Irene Pericot-Valverde ${ }^{2}$, Kaileigh Byrne ${ }^{2}$, Alain Litwin ${ }^{3}$, and Estate Sokhadze ${ }^{4}$ \\ ${ }^{1}$ Furman University and Prisma Health Upstate, Greenville, South Carolina, USA \\ ${ }^{2}$ Clemson University, Clemson, South Carolina, USA \\ ${ }^{3}$ Prisma Health-Upstate Memorial Hospital, Greenville, South Carolina, USA \\ ${ }^{4}$ University of South Carolina School of Medicine, Greenville, South Carolina, USA}

Background. Opioid use disorder (OUD) is a major public health problem in the United States that is expected to continue to increase (Blau, 2017; Hadland et al., 2018; O'Donnell et al., 2017). Maintenance treatment with buprenorphine in medication-assisted treatment (MAT) has been associated with reductions in opiate use in individuals with OUD. However, despite low physical symptoms of withdrawal, buprenorphine-treated opioid-dependent patients still demonstrate vulnerability to relapse (Hyman, 2005; Robinson \& Berridge, 2001). Craving and attentional bias towards drug-related items may contribute to the high rates of noncompliance and relapse in OUD individuals undergoing MAT. The cue-reactivity paradigm (Carter \& Tiffany, 1999) has been among the most prominent methods for investigating drug craving and psychophysiological responses to drug cues.

Purpose. We propose that craving and excessive reactivity to drug-related cues could be considered the core mechanisms underlying relapse risk. Comparing central and autonomic nervous systems activity profiles and correlates of craving, drug-cue responsiveness, and more general emotional processes may provide more knowledge towards the comprehension of the interaction between craving, affect-related states, motivational processes, and clinical outcomes of OUD patients enrolled in MAT.
Methods. In a pilot study were recruited 10 participants from outpatient individuals being treated with MAT for opioid addiction. Drug screens were conducted using saliva drug test and eligibility was confirmed by clinical and behavioral evaluations. Pictorial cue reactivity test was conducted using exposure to emotionally neutral pictures from the International Affective Picture System (IAPS; Lang et al., 2001) and drug-related images matching IAPS - pictures by color, size and background. The study used blocked design of presentation (16 images per block, $3 \mathrm{~s}$ per image) and each block was followed by subjective rating of craving. Responses were recorded with Nexus-10 psychophysiological monitor with BioTrace+ software (Mind Media, BV, The Netherlands). Photoplethysmogram (PPG), pneumogram, and electrodermal activity were acquired to measure skin conductance level (SCL), time domain heart rate variability (HRV) measures (RMSSD, SDNN), and respiration rate and amplitude. EEG was recorded from four frontal sites referenced to linked earlobes.

Results. Analysis of the frontal EEG conducted to assess power of slow (theta, alpha) and fast (high beta, gamma) rhythms in neutral and drug blocks showed higher relative power of gamma $(35-45 \mathrm{~Hz})$, along with trends to lower power of theta activity and higher alpha-to-theta ratio in response to drug cues. Autonomic responses to drug cues were featured by increased SCL, higher frequency and lower amplitude of respiration, and increased RMSSD and SDNN measures of HRV with the tendency to phasic heart rate deceleration. This psychophysiological response profile can be considered as indicative of increased attention.

Conclusions. Psychophysiological indices of heightened arousal and attention to drug cues were found to be useful objective measures to complement subjective reports of craving. Craving, cue-reactivity, attentional biases are important clinical precipitants of relapse in and their measures may serve as useful objective outcomes of behavioral therapies. Furthermore, they could be used for guiding better targeted behavioral interventions.

Acknowledgements. The study was supported by Prisma Health Seed Grant.

\section{References}

Blau, M. (2017). STAT forecast: Opioids could kill nearly 500,000 Americans in the next decade. https://www.statnews.com /2017/06/27/opioid-deaths-forecast/ 
Carter, B. L., \& Tiffany, S. T. (1999). Meta-analysis of cuereactivity in addiction research. Addiction, 94(3), 327-340.

Fatseas, M., Denis, C., Massida, Z., Verger, M., FranquesRénéric, P., \& Auriacombe, M. (2011). Cue-induced reactivity, cortisol response and substance use outcome in treated heroin dependent individuals. Biological Psychiatry, 70(8), 720-727. https://doi.org/10.1016/j.biopsych.2011.05.015

Hadland, S. E., Bagley, S. M., Rodean, J., Silverstein, M., Levy, S., Larochelle, M. R., Samet, J. H., \& Zima, B. T. (2018). Receipt of timely addiction treatment and association of early medication treatment with retention in care among youths with opioid use disorder. JAMA Pediatrics, 172(11), 10291037. https://doi.org/10.1001/jamapediatrics.2018.2143

Hyman, S. E. (2005). Addiction: A disease of learning and memory. The American Journal of Psychiatry, 162(8), 14141422. https://doi.org/10.1176/appi.ajp.162.8.1414

Lang, P. J., Bradley, M. M., \& Cuthbert, B. N. (2001) International Affective Picture System (IAPS): Instruction manual and affective ratings. Gainesville, FL: The Center for Research in Psychophysiology, University of Florida.

O'Donnell, J. K., Gladden, R. M., \& Seth, P. (2017). Trends in deaths involving heroin and synthetic opioids excluding methadone, and law enforcement drug product reports, by census region - United States, 2006-2015. MMWR Morbidity and Mortality Weekly Report, 66(34), 897-903. https://doi.org $/ 10.15585 / \mathrm{mmwr} . \mathrm{mm} 6634 \mathrm{a} 2$

Robinson, T. E., \& Berridge, K. C. (2001). Incentive-sensitization and addiction. Addiction, 96(1), 103-114. https://doi.org /10.1046/j.1360-0443.2001.9611038.x

\section{Navigating Virtual Neurofeedback Treatment During COVID-19: A Retrospective Analysis James Spears and Cerise Edmonds \\ University of Texas at San Antonio, San Antonio, Texas, USA}

Neurofeedback, or electroencephalography (EEG) biofeedback, is a therapeutic approach that integrates a client's brainwave activity to enhance and empower their specific, individualized growth through self-regulation (Demos, 2005). This modality continues to be applied to decreasing or alleviating symptoms associated with a variety of mental health concerns, including anxiety and posttraumatic stress disorder (PTSD; Gregory et al., 2020; Mennella et al., 2017; Romero et al., 2020). Traditionally, clinicians conduct neurofeedback services in person. Physical presence allows clinicians the ability to assist with certain tasks like correct electrode placement as well as mitigate any questions or technical issues that arise.

On January 30, 2020, the World Health Organization declared a public health emergency in response to the viral COVID-19 outbreak. Shortly after, the United States acknowledged a national emergency (9994Executive Office of the President, 2020), causing many colleges and universities to swiftly transition solely to online platforms (Murphy et al., 2020). Because of this unprecedented transition to virtual learning, collegiate programs that offer or require student internships as course credit were tasked with developing creative alternatives.

The counseling department at the University of Texas at San Antonio offers students the unique opportunity to complete an introductory-level and two advanced practicum neurofeedback courses, intentionally designed to fulfill most requirements for certification through the Biofeedback Certification International Alliance. Each course contains a hands-on component, ranging from weekly class labs to conducting no-cost neurofeedback sessions with clients from the community. With the transition to virtual learning regarding the pandemic, these specific classes were challenged with discovering avenues that continued to provide kinesthetic learning for students. Fortunately, through collaboration and donations from practitioners and organizations, the department's neurofeedback program offered virtual, distance neurofeedback services to clients that adhered to ethical guidelines postulated by the American Counseling Association (American Counseling Association, 2014). Neurofeedback equipment and laptop computers outfitted with BioExplorer software were distributed to each participant, while clinicians were able to access and operate client computers from the leisure of their homes.

If accepted, the poster presentation will illustrate a retrospective analysis of results and demographics gathered from the client's $(N=17)$ twice-weekly virtual sessions during the summer academic term of 2020. Those served stated having negative symptoms associated with either anxiety, PTSD, or attention-deficit/hyperactive disorder. Individualized treatment protocols were developed using preliminary theta/beta power ratio data collected before scheduled neurofeedback sessions commenced (Hernández et al., 2016; van Son et al., 2020). Presenters will also discuss the unique advantages and limitations relating to providing virtual, online neurofeedback services.

\section{References}

American Counseling Association. (2014). 2014 ACA code of ethics. https://www.counseling.org/docs/default-source /default-document-library/2014-code-of-ethicsfinaladdress.pdf

Demos, J. N. (2005). Getting started with neurofeedback. W.W. Norton.

Executive Office of the President. (2020). Proclamation 9994: Declaring a national emergency concerning the novel coronavirus disease (COVID19) outbreak. Federal Registrar, 15337-15338. https://www.federalregister.gov/documents /2020/03/18/2020-05794/declaring-a-national-emergencyconcerning-the-novel-coronavirus-disease-covid-19-outbreak 
Gregory, J. C., Romero, D. E., \& Jones, M. S. (2020). Predictors of neurofeedback outcomes following qEEG individualized protocols for anxiety. NeuroRegulation, 7(1), 18-25. https://doi.org/10.15540/nr.7.1.18

Hernández, E. D., Marqués, J. G., \& Alvarado, J. M. (2016). Effect of the theta-beta neurofeedback protocol as a function of subtype in children diagnosed with attention deficit hyperactivity disorder. The Spanish Journal of Psychology, 19, E30. https://doi.org/10.1017/sjp.2016.31

Jones, M. S., \& Hitsman, H. (2018). QEEG-guided neurofeedback treatment for anxiety symptoms. NeuroRegulation, 5(3), 8592. https://doi.org/10.15540/nr.5.3.85

Mennella, R., Patron, E., \& Palomba, D. (2017). Frontal alpha asymmetry neurofeedback for the reduction of negative affect and anxiety. Behaviour Research and Therapy, 92(1), 32-40. https://doi.org/10.1016/j.brat.2017.02.002

Murphy, L., Eduljee, N. B., \& Croteau, K. (2020). College student transition to synchronous virtual classes during the COVID-19 pandemic in northeastern United States. Pedagogical Research, 5(4). https://doi.org/10.29333/pr/8485

Romero, D. E., Anderson, A., Gregory, J. C., Potts, C. A., Jackson, A., Spears, J. R., Jones, M. S., \& Speedlin, S. (2020). Using neurofeedback to lower PTSD symptoms. NeuroRegulation, 7(3), 99-106. https://doi.org/10.15540 /nr.7.3.99

van Son, D., van der Does, W., Band, G. P. H., \& Putman, P. (2020). EEG theta/beta ratio neurofeedback training in healthy females. Applied Psychophysiology and Biofeedback, 45, 195-210. https://doi.org/10.1007/s10484-020-09472-1

\section{Neurofeedback and Trauma \\ Melanie Gardner \\ The Creative Arts Space, LLC, Petersburg, Virginia, USA}

Traumatic events do not discriminate and can occur to anyone. The aftermath of the trauma impacts not only the victim but family, friends, coworkers, and their community as well. The numbers are staggering with the PTSD Alliance (2018) reporting that over 13 million or $5 \%$ of Americans suffer from PTSD during any moment in time, while the yearly prevalence was reported to be $3.6 \%$. The financial costs of anxiety disorders, including PTSD, amount to well over $\$ 42$ billion with many of these dollars being spent on repetitive healthcare visits due to inaccurate diagnoses (PTSD Alliance, 2018).

This qualitative systematic literature review will focus on the current research on quantitative electroencephalography (qEEG), neurofeedback assessment and neurofeedback training with individuals who have experienced psychological trauma. The analysis of the literature surrounding neurofeedback assessment and training will also address the feasibility of identifying the area or areas of the brain impacted by psychological trauma, as well as the magnitude of the impact. Finally, it will attempt to identify areas of the brain that may benefit from training if they were altered by trauma. The overarching goal of the study is to determine if using neurofeedback assessment and training can aid in reducing the time and costs involved in establishing an accurate diagnosis, and ultimately, returning the individual to an increased level of functioning.

The statement of the problem section will identify the high cost of trauma for the individual, family, and society. The purpose of the study is to analyze the literature on neurofeedback assessment and training with individuals who have experienced psychological trauma, identify the psychological impact on the brain, and the manner in which neurofeedback assessment and training may accurately diagnose trauma, facilitate early treatment and decrease the cost of trauma to all involved. The theoretical framework of adaptive information processing theory will provide the lens through which the existing research and findings of the study will be viewed.

Finally, the significance of this qualitative systematic literature review, as well as limitations and delimitations are identified. Key words utilized include neurofeedback, EEG biofeedback, psychological trauma, posttraumatic stress disorder, PTSD, acute stress disorder, electroencephalography, EEG, quantitative EEG, QEEG, brain map, brain computer interface.

Participants were adult individuals with a history of trauma ranging from single incident trauma to chronic and developmental trauma. Findings are revealed and implications for the profession and field are noted.

\section{References}

Dondanville, K. A., Borah, E. V., Bottera, A. R., \& Molino, A. T. (2018). Reducing stigma in PTSD treatment seeking among service members: Pilot intervention for military leaders. Best Practices in Mental Health, 14(1), 15-26.

Feng, P., Becker, B., Feng, T., \& Zheng, Y. (2018). Alter spontaneous activity in amygdala and vmPFC during fear consolidation following $24 \mathrm{~h}$ sleep deprivation. Neurolmage, 172, 461-469. https://doi.org/10.1016 /j.neuroimage.2018.01.057

Fisher, S. F., Lanius, R. A., \& Frewen, P. A. (2016). EEG neurofeedback as adjunct to psychotherapy for complex developmental trauma-related disorders: Case study and treatment rationale. Traumatology, 22(4), 255-260. https://doi.org/10.1037/trm0000073

Gapen, M., van der Kolk, B. A., Hamlin, E., Hirshberg, L., Suvak, M., \& Spinazzola, J. (2016). A pilot study of neurofeedback for chronic PTSD. Applied Psychophysiology and Biofeedback, 41, 251-261. https://doi.org/10.1007/s10484015-9326-5

Meiser-Stedman, R., McKinnon, A., Dixon, C., Boyle, A., Smith, P., \& Dalgleish, T. (2017). Acute stress disorder and the transition to posttraumatic stress disorder in children and adolescents: Prevalence, course, prognosis, diagnostic suitability, and risk markers. Depression and Anxiety, 34(4), 348-355. https://doi.org/10.1002/da.22602 
Peniston, E. G., \& Kulkosky, P. J. (1991). Alpha-theta brainwave neuro-feedback for Vietnam veterans with combat-related post-traumatic stress disorder. Medical Psychotherapy, 4, 4760.

Perry, B. D. (2008). Child maltreatment: A neurodevelopmental perspective on the role of trauma and neglect in psychopathology. In T. Beauchaine, \& S. P. Hinshaw (Eds.), Child and adolescent psychopathology (pp. 93-129). John Wiley \& Sons.

Rogala, J., Jurewicz, K., Paluch, K., Kublik, E., Cetnarski, R., \& Wróbel, A. (2016). The do's and don'ts of neurofeedback training: A review of the controlled studies using healthy adults. Frontiers in Human Neuroscience, 10, 301. https://doi.org/10.3389/fnhum.2016.00301

van der Kolk, B., Ford, J. D., \& Spinazzola, J. (2019). Comorbidity of developmental trauma disorder (DTD) and post-traumatic stress disorder: Findings from the DTD field trial. European Journal of Psychotraumatology, 10(1). https://doi.org/10.1080 /20008198.2018.1562841

van der Kolk, B. A., Hodgdon, H., Gapen, M., Musicaro, R., Suvak, M. K., Hamlin, E., \& Spinazzola, J. (2016). A randomized controlled study of neurofeedback for chronic PTSD. PLOS ONE, 11(12). https://doi.org/10.1371 /journal.pone.0166752

\section{Improving Mental Health Through Z-score} LORETA Neurofeedback During a Pandemic Ingrid Valentin and Robin van Osch

NeuroVP V O F, Maastricht, Limburg, Netherlands

During the COVID-19 pandemic, the general population's mental health has been decreasing around the world. This creates a high need to find efficacious methods to improve mental health. Pharmacotherapy can lead to side effects and its effect subsides when the treatment is withdrawn. The field of neurofeedback, or EEG biofeedback, is over 50 years old. It has proven its efficacy in a wide range of psychological and cognitive symptoms. LORETA Z-score neurofeedback training (LZNFB) is a relatively new advancement in this field and, as such, there is a limited body of evidence investigating its efficacy. The aim of this study is to evaluate its effect in an uncontrolled clinical setting.

Participants. Thirty-one adult clients $(21$ men, 10 women, mean age $M=31.63, S D=9.5$ ) with mental health complaints filled in the Brief Symptom Inventory (BSI-53) before and after their neurofeedback therapy. Nine clients also responded to a follow-up questionnaire 3 months later.

Method. All clients started with an intake and qEEG assessment on which their LZNFB protocol was based. All qEEG analyses and protocols were executed and designed by one lead clinician. Before the first training session patients filled out the BSI-53 in order to determine symptoms related to the physiological profile. Each LZNFB session lasted between 30 and 40 minutes. A training protocol was used for at least five sessions before possibly altering it. The training protocol was designed to target a known neural network, that has a significant role in the client's symptomatology. Since this study was made in a clinical setting and received no funding, each client did a number of sessions that was estimated to be the best compromise between clinical efficacy and the possibilities of their financial situation. After their last session, they filled the BSI53 again. A follow-up BSI-53 measurement was requested 12 weeks after the last session.

Results. The participants did 16 sessions on average $(M=16.16, S D=6.11)$. On average, the BSI-53 scores after neurofeedback $(M=46.16, S E=$ 5.77) were lower than before neurofeedback $(M=$ $71.71, S E=6.01)$. This difference, $25.55, \mathrm{BCa} 95 \%$ $\mathrm{Cl}[17.43,33.67]$, was significant, $t(30)=6.42, p<$ .001 , and represented a medium to large effect size of $d=.76$. Three months after the last session, the scores were still lower than before neurofeedback $(M=42.11, S E=10.31)$, which was also significant $t(8)=4.54, p=.002$, and represented a large effect size of $d=.84$. Three out of $31(9.6 \%)$ participants were classified as nonresponders (less than 5\% change in scores). The only noticeable side effect was fatigue, which subsided within a day after each session.

Conclusion. LZNFB shows promise to improve pandemic-related and unrelated mental health conditions, with a high response rate, potentially long-term health outcomes and side effects limited to a short-lived fatigue. Because of the clinical, uncontrolled design of the study, mitigating factors were difficult to exclude. For example, nine participants changed their psychoactive medication habits during their neurofeedback sessions. Controlled studies are needed in order to replicate effect sizes and confirm the significance of these results.

\section{References}

Drobnjak, S. (2013). Brief Symptom Inventory. In M. D. Gellman \& J. R. Turner (Eds.), Encyclopedia of behavioral medicine (pp. 269-270). New York, NY: Springer. https://doi.org/10.1007 1978-1-4419-1005-9_3

Duric, N. S., Assmus, J., Gundersen, D., \& Elgen, I. B. (2012) Neurofeedback for the treatment of children and adolescents with ADHD: A randomized and controlled clinical trial using parental reports. BMC Psychiatry, 12, 107. https://doi.org /10.1186/1471-244X-12-107

Evans, J. R., Dellinger, M. B., \& Russell, H. L. (2020). Neurofeedback: The first fifty years. Academic Press.

Koberda, J. L. (2015) LORETA Z-score neurofeedbackeffectiveness in rehabilitation of patients suffering from traumatic brain injury. Journal of Neurology and Neurobiology, 1(4). https://doi.org/10.16966/2379-7150.113 
Koberda, J. L., Koberda, P., Moses, A., Winslow, J., Bienkiewicz, A., \& Koberda, L. (2014). Z-Score LORETA neurofeedback as a potential therapy for ADHD. Biofeedback, 42(2), 74-81. https://doi.org/10.5298/1081-5937-42.2.05

Thatcher, R. W. (2013). Latest developments in live Z-score training: Symptom check list, phase reset, and LORETA Zscore biofeedback. Journal of Neurotherapy, 17(1), 69-87. https://doi.org/10.1080/10874208.2013.759032

Thatcher, R. W., Lubar, J. F. (2015). Z score neurofeedback: Clinical applications. Academic Press.

Thatcher, R. W., North, D., \& Biver, C. (2005). Evaluation and validity of a LORETA normative EEG database. Clinical EEG and Neuroscience, 36(2), 116-122 https://doi.org/10.1177 $/ 155005940503600211$

Thatcher, R. W., Walker, B. A., Biver, C. J., North, M. A., \& Curtin, R. (2003). Sensitivity and specificity of an EEG database, validation and clinical correlation. Journal of Neurotherapy, 7(3/4), 87-121.

Wigton, N. L. (2013). Clinical perspectives of 19-channel Z-score neurofeedback: Benefits and limitations. Journal of Neurotherapy, 17(4), 259-264. https://doi.org/10.1080 /10874208.2013.847142

Wigton, N., \& Krigbaum, G. (2015). A review of qEEG-guided neurofeedback. NeuroRegulation, 2(3), 149-155. https://doi.org/10.15540/nr.2.3.149

\section{Comparing the EEG Patterns Between Patients with Major Depressive Disorder and Healthy Adults Through a Normalized Database in \\ Taiwan \\ Yin-Chen Wu and I-Mei Lin \\ Kaohsiung Medical University, Kaohsiung City, Kaohsiung, \\ Taiwan}

Background. Brain hyperactivity has been confirmed as a trait marker in patients with major depressive disorder (MDD). Several quantitative electroencephalography (qEEG) databases are available in the United States, patients' EEG data were compared with the QEEG database and transformed into the $z$-score. However, Taiwan has not yet established a qEEG database in healthy populations. This study aimed to collect and develop the qEEG database of healthy adults and compared the qEEG patterns between patients with MDD and healthy adults.

Method. This study was based on the EEG collection and development of the qEEG database. All of participants received Beck Depression Inventory-II and Beck Anxiety Inventory. The 19channel EEG raw signals were recorded for $5 \mathrm{~min}$ resting with eyes closed. NeuroGuide software was used to analyze 184 healthy adults, divided into 10year age groups from 20 to 70 , and calculated the mean and standard deviation of each age group. The statistical analysis was converted 146 raw EEG data from patients with MDD into the $z$-scores.
Results. (1) Patients with MDD revealed higher absolute high-beta at prefrontal lobe (F3/F4), parietal lobe (P3/P4), and midline (Fz/Cz/Pz) compared with the healthy adults. (2) The average $z$-score in high-beta and the absolute value of $z$ score in high-beta were both greater than 1 at prefrontal lobe (F3/F4), parietal lobe (P3/P4), and midline $(\mathrm{Fz} / \mathrm{Cz} / \mathrm{Pz})$ in patients with MDD. (3) There were positive correlations between depression and the absolute high-beta of $z$-score at F3, P3, P4, Cz, $\mathrm{Pz}$; as well as positive correlations between anxiety and the absolute high-beta of $z$-score at F4, P3, P4, Pz.

Conclusion. The brain hyperactivity was confirmed in the high-beta frequency band in patients with MDD. Moreover, the average $z$-score was greater than 1 , and the $z$-score of high-beta was correlated with depression and anxiety. This study indicated that the $z$-score can be used as a reference standard for qEEG in patients with MDD.

\section{References}

Coutin-Churchman, P., Añez, Y., Uzcátegui, M., Alvarez, L., Vergara, F., Mendez, L., \& Fleitas, R. (2003). Quantitative spectral analysis of EEG in psychiatry revisited: Drawing signs out of numbers in a clinical setting. Clinical Neurophysiology, 114(12), 2294-2306. https://doi.org /10.1016/S1388-2457(03)00228-1

Fingelkurts, A. A., Fingelkurts, A. A., Rytsälä, H., Suominen, K., Isometsä, E., \& Kähkönen, S. (2006). Composition of brain oscillations in ongoing EEG during major depression disorder. Neuroscience Research, 56(2), 133-144. https://doi.org /10.1016/j.neures.2006.06.006

Grin-Yatsenko, V. A., Baas, I., Ponomarev, V. A., \& Kropotov, J. D. (2009). EEG power spectra at early stages of depressive disorders. Journal of Clinical Neurophysiology, 26(6), 401406. https://doi.org/10.1097/WNP.0b013e3181c298fe

Johnstone, J., \& Gunkelman, J. (2003). Use of databases in QEEG evaluation. Journal of Neurotherapy, 7(3-4), 31-52. https://doi.org/10.1300/J184v07n03_02

Lee, T. W., Yu, Y. W.-Y., Chen, M.-元., \& Chen, T.-J. (2011). Cortical mechanisms of the symptomatology in major depressive disorder: A resting EEG study. Journal of Affective Disorders, 131(1-3), 243-250. https://doi.org/10.1016 /j.jad.2010.12.015

Lin, I.-M., Chen, T.-C., Lin, H.-Y., Wang, S.-Y., Sung, J.-L., \& Yen, C.-W. (2021). Electroencephalogram patterns in patients comorbid with major depressive disorder and anxiety symptoms: Proposing a hypothesis based on hypercortical arousal and not frontal or parietal alpha asymmetry. Journal of Affective Disorders, 282, 945-952. https://doi.org/10.1016 /j.jad.2021.01.001

Lorensen, T. D., \& Gow, K. M. (2003). Quantitative electroencephalographic comodulation: An investigation of patterns in chronic fatigue syndrome. Journal of Neurotherapy, 7(1), 3-18. https://doi.org/10.1300 IJ184v07n01_02

Newson, J. J. \& Thiagarajan, T. C. (2019). EEG frequency bands in psychiatric disorders: A review of resting state studies. Frontiers in Human Neuroscience, 12, 521. https://doi.org /10.3389/fnhum.2018.00521 
Prichep, L. S. (2005). Use of normative databases and statistical methods in demonstrating clinical utility of QEEG: Importance and cautions. Clinical EEG and Neuroscience, 36(2), 82-87. https://doi.org/10.1177/155005940503600207

Thatcher, R. W., \& Lubar, J. F. (2009). History of the scientific standards of QEEG normative databases. In T. H. Budzynski, H. K. Budzynski, J. R. Evans, \& A. Abarbanel (Eds.), Introduction to quantitative EEG and neurofeedback:
Advanced theory and applications (pp. 29-59). Academic Press. https://doi.org/10.1016/B978-0-12-374534-7.00002-2

Received: December 12, 2021

Accepted: December 12, 2021

Published: December 31, 2021 